\title{
The Combination of Cyst Fluid Carcinoembryonic Antigen, Cytology and Viscosity Increases the Diagnostic Accuracy of Mucinous Pancreatic Cysts
}

\author{
Se Hun Oh ${ }^{1}$, Jong Kyun Lee ${ }^{1}$, Kyu Taek Lee ${ }^{1}$, Kwang Hyuck Lee ${ }^{1}$, Young Sik Woo ${ }^{2}$, and Dong Hyo Noh ${ }^{1}$ \\ ${ }^{1}$ Division of Gastroenterology, Department of Medicine, Samsung Medical Center, Sungkyunkwan University School of Medicine, Seoul, and \\ ${ }^{2}$ Division of Gastroenterology, Department of Internal Medicine, Wonkwang University Sanbon Hospital, Wonkwang University College of \\ Medicine, Gunpo, Korea
}

Background/Aims: The objective of this study was to investigate the value of cyst fluid carcinoembryonic antigen (CEA) in combination with cytology and viscosity for the differential diagnosis of pancreatic cysts. Methods: We retrospectively reviewed our data for patients who underwent endoscopic ultrasound-guided fine needle aspiration (EUS-FNA) and cyst fluid analysis. We investigated the sensitivity, specificity and accuracy of the combination of cyst fluid CEA, cytology and viscosity testing. Results: A total of 177 patients underwent EUS-FNA and cyst fluid analysis. Of these, 48 subjects were histologically and clinically confirmed to have pancreatic cysts and were therefore included in the analysis. Receiver operator curve analysis demonstrated that the optimal cutoff value of cyst fluid CEA for differentiating mucinous versus nonmucinous cystic lesions was $48.6 \mathrm{ng} / \mathrm{mL}$. The accuracy of cyst fluid CEA $(39 / 48,81.3 \%)$ was greater than the accuracy of cytology $(23 / 45,51.1 \%)$ or the string sign (33/47, 70.2\%). Cyst fluid CEA in combination with cytology and string sign assessment exhibited the highest accuracy (45/48, 93.8\%). Conclusions: Cyst fluid CEA was the most useful single test for identifying mucinous pancreatic cysts. The addition of cytology and string sign assessment to cyst fluid CEA increased the overall accuracy for the diagnosis of mucinous pancreatic cysts. (Gut Liver 2017;11:283-289)

Key Words: Pancreatic cyst; Cyst fluid analysis; Cyst fluid carcinoembryonic antigen

\section{INTRODUCTION}

In recent years, detection of pancreas cystic mass has increased by widespread use of imaging studies. ${ }^{1-3}$ Cystic lesions of pancreas can be divided into mucinous and nonmucinous cyst. Mucinous cysts are classified into intraductal papillary mucinous neoplasm (IPMN) and mucinous cystic neoplasm (MCN) by World Health Organization classification 2000. ${ }^{4}$ Because these mucinous cysts (MCN and IPMN) are considered premalignant lesions, it is important to distinguish mucinous from nonmucinous cysts to select treatment options when pancreas cystic lesions are detected. Standard imaging methods such as transabdominal ultrasound, computed tomography (CT), and magnetic resonance imaging (MRI) are often used to differentiate the two. However, their diagnostic accuracies are not satisfactory. ${ }^{5-8}$ Thus, a need for other methods has emerged.

Recently, many studies reported about cyst fluid analysis, cytology, and viscosity test by endoscopic ultrasonography (EUS) and EUS-guided fine needle aspiration (FNA) for differential diagnosis of pancreatic cyst. However, single use of each method had its limitations and there were few reports about combination of these methods. So the object of this study was to determine the accuracy of combination test of the three methods for differential diagnosis of mucinous versus nonmucinous pancreas cystic lesions.

\section{MATERIALS AND METHODS}

\section{Subjects and EUS procedures}

We investigated Samsung Medical Center database to identify patients who underwent EUS-FNA and cyst fluid analysis due to pancreas cystic lesions that could not be definitely diagnosed on CT or MRI between January 2008 and May 2014 in one tertiary referral center in Korea. The study was approved by Institutional Review Board of our institution. Patient consent for data collection was waived. Pathologic reports of resected cystic lesions

Correspondence to: Jong Kyun Lee

Division of Gastroenterology, Department of Medicine, Samsung Medical Center, Sungkyunkwan University School of Medicine, 81 Irwon-ro, Gangnam-gu, Seoul 06351, Korea

Tel: +82-2-3410-3409, Fax: +82-2-3410-6983, E-mail: jongk.lee@samsung.com

Received on December 26, 2015. Revised on April 14, 2016. Accepted on April 14, 2016. Published online September 9, 2016 pISSN 1976-2283 eISSN 2005-1212 https://doi.org/10.5009/gnl15650

() This is an Open Access article distributed under the terms of the Creative Commons Attribution Non-Commercial License (http://creativecommons.org/licenses/by-nc/4.0) which permits unrestricted non-commercial use, distribution, and reproduction in any medium, provided the original work is properly cited. 
were investigated. They were classified as mucinous cystic lesion (MCN, IPMN, benign, or malignant) or nonmucinous cystic lesion including serous, or inflammatory cyst. Cystic lesions that could not be classified into the categories stated above were classified as others. Results of EUS imaging, cytology, cyst fluid carcinoembryonic antigen (CEA) were collected. Viscosity measured by maximal length of mucus string between examiner's thumb and index finger were collected and compared to histology or typical clinical features of pseudocyst as the final diagnostic standard. Endoscopic ultrasound examination were performed using a linear scanning echoendoscope (GF-UCT240; Olympus Co., Tokyo, Japan) with ultrasound observation systems (ALOKA Prosound alpha-5 or F-75; Aloka Co., Ltd., Tokyo, Japan). On EUS report, location, size, and morphology of cystic lesion were collected. The morphology of the cystic lesion by EUS was investigated. Specific morphologic findings were recorded, including shape, loculation, presence or absence of echoic internal content, septation, wall thickening, mural nodule, daughter cyst, calcification, communication with pancreatic duct (PD), and PD dilatation. Results of string sign was described by examiners. Cystic lesions were aspirated under EUS guidance using 25-, 22-, or 19-gauge needle (Echo-Tip; WilsonCook Medical Inc., Winston-Salem, NC, USA) for cytology and cyst fluid analysis. A 25-gauge needle was used in one case. The 19 -gauge needles were used in four cases. The 22-gauge needles were used in other 43 cases. Cytology reports were investigated. The diagnosis of mucinous cystic lesion by cytology was defined as containing cytologic evidence of mucinous epithelium (clusters of columnar epithelial cells with cytoplasmic mucin). Aspirated fluid was centrifuged and supernatant was subjected to CEA measurement using radioimmunoassay method.

\section{Data collection and analysis}

Analyses were performed for patients with histologic confirmation of the type of cystic lesion or clinically confirmed as pseudocyst. Statistical analysis was performed by using chisquare or Fisher exact tests for categorical variables. The Student t-test or Mann-Whitney test was used for continuous variables. Receiver operator characteristic (ROC) curve was plotted using cyst fluid CEA to predict mucinous cystic lesion. The area under ROC curve, a measure of predictive power, was calculated. Cutoff value was selected to maximize the proportion of correct classifications of the cystic lesion. The sensitivity, specificity, and accuracy rate (percentage) of cyst fluid CEA were calculated using this cutoff value. The sensitivity, specificity, and accuracy rate of cytology, string sign and combinations of these tests were also measured. Statistical analysis was executed using SAS version 9.3 (SAS Institute Inc., Cary, NC, USA). Statistical significance was considered when p-value was less than 0.05.

\section{RESULTS}

\section{Classification and basic characteristics of pancreatic cysts}

A total of 177 patients who had indeterminate pancreatic cyst lesion on CT or MRI image underwent EUS-FNA and cyst fluid analysis. Forty-three of these patients underwent surgical resection, which provided histologic diagnosis of cystic lesions, including 16 MCNs, 13 IPMNs, six serous cystic neoplasms (SCNs), four pseudocysts, and four others (rare cystic lesions, including two cystic neuroendocrine tumors, one ancient schwannoma, and one squamoid cyst). The remaining 134 patients were followed up without operation. Among these followed up subjects, nine patients were clinically defined as pseudocyst and they

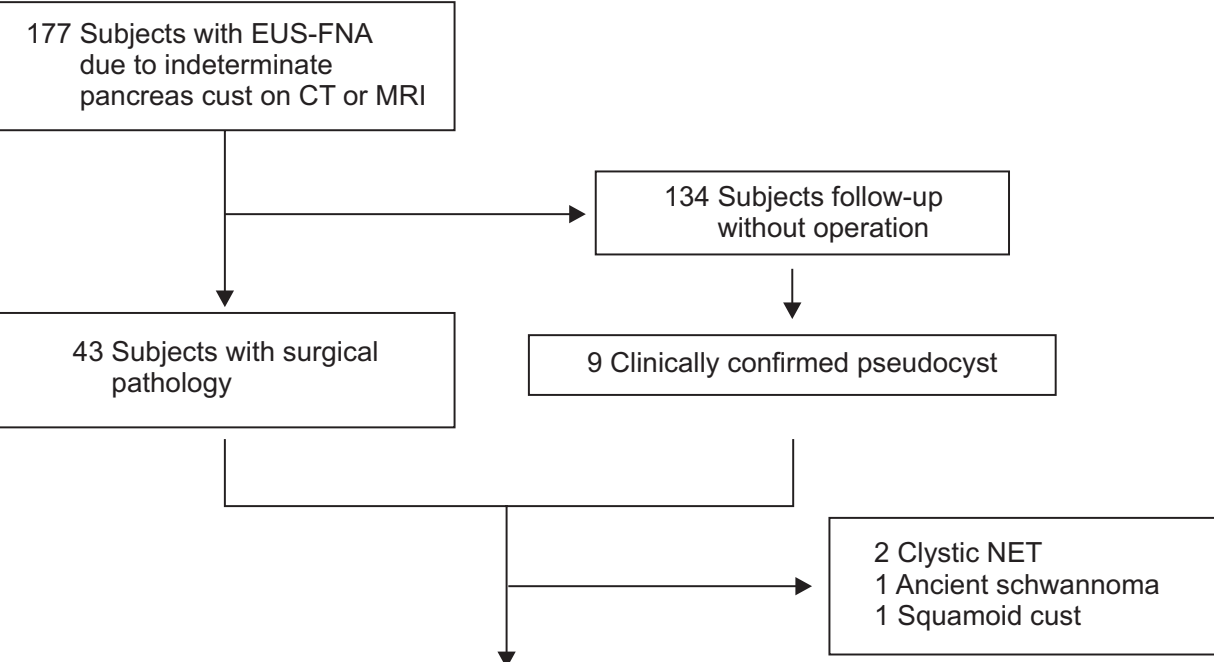

48 Subjects $\{$ Mucinous (16 MCN and 13 IPMN) Nonmucinous (6 SCN and 13 pseudocyst)
Fig. 1. Flow chart for patient selection.

EUS-FNA, endoscopic ultrasoundguided fine needle aspiration; CT, computed tomography; MRI, magnetic resonance imaging; NET, neuroendocrine tumor; $\mathrm{MCN}$, mucinous cystic neoplasm; IPMN, intraductal papillary mucinous neoplasm; SCN, serous cystic neoplasm. 
were included to selected subjects. Clinically confirmed pseudocyst was defined when satisfying all of the following conditions: history of pancreatitis, no neoplastic cell and inflammatory cells only on cytology, and disappearance and no recurrence of cyst during 1 year of follow-up. Finally, a total of 48 subjects were selected except the four rare cystic lesions (others) (Fig. 1). Basic characteristics of patients are summarized in Table 1 . There was no significant difference in mean age, sex, location of cyst, or mean cyst size between the mucinous cyst group and the nonmucinous cyst group. Male was dominant in IPMN and pseudocyst groups than in MCN and serous cystic neoplasm groups. Cysts were dominant in tail in MCN and pseudocyst groups than in IPMN and SCN groups. Mean cyst size of IPMN was smaller than other groups. Most examined cysts (46/48, 95.8\%) were greater than $2 \mathrm{~cm}$ in diameter. Most (26/29, 89.7\%) mucinous lesions were benign. Only three of 29 (10.3 \%) were malignant.

\section{Results of EUS morphology}

As for EUS findings, there was no statistically significant difference between the mucinous cyst group and the nonmucinous cyst group. Presence of multiloculation, wall thickening, septation, and PD dilatation were significantly $(\mathrm{p}<0.05)$ different EUS features between some groups. Daughter cyst was observed only in the MCN group. Echoic internal content and surrounding pa- renchymal change in the pseudocyst group and PD communication with cyst in the IPMN group were statistically significant $(p<0.05)$ EUS features compared to other groups (Table 2).

\section{Cyst fluid CEA}

The range of mucinous cyst fluid CEA was from 0.6 to 43,170 $\mathrm{ng} / \mathrm{mL}$. The range of nonmucinous cyst fluid CEA was from 0.3 to $56.27 \mathrm{ng} / \mathrm{mL}$. The mean cyst fluid CEA concentration of mucinous cysts $(2,893.4 \mathrm{ng} / \mathrm{mL})$ was greater than the mean cyst fluid CEA concentration of all nonmucinous cystic lesions (7.8 $\mathrm{ng} / \mathrm{mL}$ ). Mucinous cysts with malignancy had greater mean cyst fluid CEA concentrations $(4,244.5 \mathrm{ng} / \mathrm{mL})$ than cyst fluid CEA concentrations of benign mucinous cysts $(2,737.5 \mathrm{ng} /$ $\mathrm{mL}$ ). However there was no statistically significant difference $(p=0.77)$. Receiver operator curve analysis of the cyst fluid CEA demonstrated that the optimal cutoff value of cyst fluid CEA for differentiating mucinous versus nonmucinous cystic lesions was $48.6 \mathrm{ng} / \mathrm{mL}$. The area under the curve was 0.87 (Fig. 2). Using this cutoff value, the sensitivity, specificity, and accuracy of cyst fluid CEA for the diagnosis of a mucinous cyst was $72.4 \%$ (21/29), 94.7\% (18/19), and 81.3\% (39/48), respectively. The sensitivity, specificity, and accuracy of cystic fluid CEA, cytology, string sign, and the combination of the three to predict whether the cysts were mucinous or nonmucinous are shown in

Table 1. Basal Characteristics of the Patients

\begin{tabular}{|c|c|c|c|c|c|c|c|c|}
\hline \multirow{2}{*}{ Characteristic } & \multirow{2}{*}{ No. } & \multirow{2}{*}{ Age, yr } & \multicolumn{2}{|c|}{ Sex } & \multicolumn{3}{|c|}{ Location } & \multirow{2}{*}{ Size, cm } \\
\hline & & & Male & Female & Head & Body & Tail & \\
\hline Mucinous & 29 & 57.6 & 15 & 14 & 7 & 8 & 14 & 4.1 \\
\hline MCN & 16 & 53.0 & 5 & 11 & 2 & 4 & 10 & 5.1 \\
\hline IPMN & 13 & 63.3 & 10 & 3 & 5 & 4 & 4 & 3.0 \\
\hline Nonmucinous & 19 & 50.8 & 13 & 6 & 8 & 2 & 9 & 5.5 \\
\hline SCN & 6 & 45.2 & 2 & 4 & 5 & 1 & 0 & 4.2 \\
\hline Pseudocyst & 13 & 53.4 & 11 & 2 & 3 & 1 & 9 & 6.1 \\
\hline
\end{tabular}

MCN, mucinous cystic neoplasm; IPMN, intraductal papillary mucinous neoplasm; SCN, serous cystic neoplasm.

Table 2. Endoscopic Ultrasonography Features of the Pancreatic Cyst Lesions

\begin{tabular}{|c|c|c|c|c|}
\hline & MCN $(n=16)$ & $\operatorname{IPMN}(\mathrm{n}=13)$ & Pseudocyst $(n=13)$ & $\mathrm{SCN}(\mathrm{n}=6)$ \\
\hline Multiloculation & $9 / 16(56.3)$ & $12 / 13(92.3)^{*}$ & $4 / 13(30.8)^{*}$ & $4 / 6(66.7)$ \\
\hline Echoic internal content & $8 / 16(50)^{*,+}$ & $1 / 13(7.7)^{* . \neq}$ & $13 / 13(100)^{\dagger, \pm, \S}$ & $2 / 6(33.3)^{\S}$ \\
\hline Wall thickening & $8 / 16(50)^{*}$ & $7 / 13(53.8)$ & $12 / 13(92.3)^{*, \pm}$ & $0^{\ddagger}$ \\
\hline Septation & $9 / 16(56.3)^{*}$ & $13 / 13(100)^{*, \pm}$ & $7 / 13(53.8)^{\ddagger}$ & $5 / 6(83.3)$ \\
\hline Daughter cyst & $5 / 16(31.3)^{* \pm} \pm$ & $0^{*}$ & $0^{\ddagger}$ & 0 \\
\hline PD communication & $1 / 16(6.3)^{*}$ & $8 / 13(61.5)^{*,+, \neq}$ & $1 / 13(7.7)^{\dagger}$ & $0^{\ddagger}$ \\
\hline PD dilatation & $1 / 16(6.3)^{*}$ & $8 / 13(61.5)^{*, \pm}$ & $3 / 13(23.1)$ & $0^{\ddagger}$ \\
\hline Surrounding parenchyme change & $1 / 16(6.3)^{*}$ & $0^{\ddagger}$ & $8 / 13(61.5)^{*, t, \pm}$ & $0^{\dagger}$ \\
\hline
\end{tabular}

Data are presented as number (\%).

MCN, mucinous cystic neoplasm; IPMN, intraductal papillary mucinous neoplasm; SCN, serous cystic neoplasm.

${ }_{*,+,+, 8}$ Means statiscally significant difference between corresponding groups. 
Table 3. The overall accuracy of CEA (81.3\%) was greater than that of cytology (51.1\%) or string sign (72.3\%). When cutoff value of $192 \mathrm{ng} / \mathrm{mL}$ reported in the largest multicenter study ${ }^{9}$ was used, the sensitivity and the accuracy decreased to $48.3 \%$ and $68.8 \%$, respectively, but the specificity increased to $100 \%$.

\section{Cytology}

Results of cytology were used to categorize cysts into mucinous or nonmucinous types. Cytology report positive for mucinous cyst was defined as the presence of columnar epithelium with mucin-containing cells or mucin and the presence of malignant cells (mucinous cystadenocarcinoma and malignant IPMN). The sensitivity, specificity of cytology for diagnosing a mucinous cyst were 24.1\% (7/29), 100\% (16/16), respectively. The accuracy was 51.1\% (23/45), which was the lowest among all tests.

\section{String sign}

String sign was checked in all cases except for one patient. Positive string sign was defined when maximal length of mucus string which was measured by ruler between examiner's thumb and index finger and it was longer than $3 \mathrm{~mm}$. Fifteen patients showed positive string sign, whereas 22 patients were string sign negative. The sensitivity, specificity, and accuracy of string

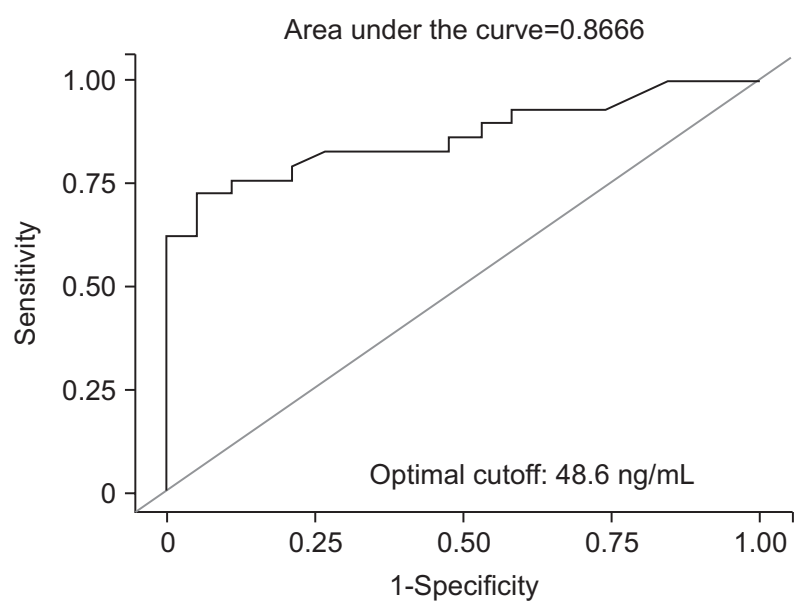

Fig. 2. Receiver operator characteristic curve of cyst fluid carcinoembryonic antigen. sign for the diagnosis of mucinous cysts were 53.6\% (15/28), 100\% (19/19), and 72.3\% (34/47), respectively. The mean cyst fluid CEA in string sign positive group was $4,573.4 \mathrm{ng} / \mathrm{mL}$. The proportion of patients with cyst fluid CEA $>48.6 \mathrm{ng} / \mathrm{mL}$ (cutoff values in this study) was $66.7 \%$ in the string sign positive group. They were greater than the mean cyst fluid CEA (482.2 $n g / m L)$ or the proportion of patients with cyst fluid CEA >48.6 $\mathrm{ng} / \mathrm{mL}(37.5 \%)$ in the string sign negative group. However, there was no statistical significance ( $\mathrm{p}=0.18$ and $\mathrm{p}=0.12$, respectively). When it was confined to mucinous cysts, the mean cyst fluid CEA of string sign positive group $(4,573.4 \mathrm{ng} / \mathrm{mL})$ was greater than that of the string sign negative group $(1,175.6 \mathrm{ng} / \mathrm{mL})$. The proportion of patients with cyst fluid CEA $>48.6 \mathrm{ng} / \mathrm{mL}$ of string sign positive group (66.7\%) was smaller than that of patients with cyst fluid CEA $>48.6 \mathrm{ng} / \mathrm{mL}$ of string sign negative group (84.6\%). However, there was no statistical significance ( $p=0.12$ and $\mathrm{p}=0.22$, respectively).

\section{Combination testing}

The combination of the three tests (cyst fluid CEA, cytology, and string sign) in identifying mucinous cysts were analyzed. In this combination, a cyst was classified as mucinous cyst if any one of the component tests was positive. The combination of cytology, string sign, and CEA was more sensitive without decreasing specificity than CEA alone ( $93.1 \%$ vs $72.4 \%, p=0.03)$. It showed increasing accuracy (93.8\% vs $81.3 \%, p=0.03$ ) (Table 3 ).

\section{DISCUSSION}

When a pancreatic cyst is detected, it is important to identify the category first, as selection of treatment options generally depend on whether it is mucinous cyst or not. For nonmucinous cysts, observation and follow-up are usually the treatment of choice unless they cause symptoms because nonmucinous cysts do not have malignant potential. In contrast, as mucinous cysts have variable malignant potential, surgical resection should be considered. For discriminating mucinous cysts from nonmucinous cysts, standard imaging test such as CT is used firstly. But overall diagnostic accuracy for such differentiation is relatively low. ${ }^{10,11}$ Since the introduction of EUS, we were able to obtain more detail informations for many different types of cysts ${ }^{12-14}$

Table 3. Sensitivity, Specificity, and Accuracy of Cytology, Cyst Fluid CEA, String Sign, or a Combination of the Three for the Diagnosis of Mucinous Cysts

\begin{tabular}{cccccc}
\hline & \multicolumn{1}{c}{ CEA $^{*}$} & Cytology & String sign & Combination $^{\dagger}$ & p-value vs $^{\dagger}$ \\
\hline Sensitivity & $21 / 29(72.4)$ & $7 / 27(24.1)$ & $15 / 28(53.6)$ & $27 / 29(93.1)$ & 0.03 \\
Specificity & $18 / 19(94.7)$ & $16 / 16(100)$ & $19 / 19(100)$ & $18 / 19(94.7)$ & NS \\
Accuracy & $39 / 48(81.3)$ & $23 / 45(51.1)$ & $34 / 47(72.3)$ & $45 / 48(93.8)$ & 0.03 \\
\hline
\end{tabular}

Data are presented as number (\%).

CEA, carcinoembryonic antigen; NS, not significant.

*Using $48.6 \mathrm{ng} / \mathrm{mL}$ as cutoff; ${ }^{\dagger}$ Combination: cytology (+) or string sign (+) or CEA (+)*. 
and some studies reported improved accuracy. ${ }^{13,15-18}$ However, in a large multicenter study, the overall accuracy of EUS morphology alone for differentiation of mucinous cyst was low only at $51 \% .^{9}$ In our study, there were some statistically significant different features (presence of multiloculation, echoic internal content, wall thickening, and septation) among some groups on EUS findings. But these features were overlapped among some groups. And there were relatively specific findings on each different types of pancreas cyst. For example, daughter cyst in MCN, echoic internal content and surrounding parenchymal change in pseudocyst, and PD communication with cyst in IPMN group. However, we failed to find definite specific findings to distinguish mucinous cysts from nonmucinous cysts. One of the reasons might be that cysts which had definite morphologic finding on $\mathrm{CT}$, for example honeycombing appearance of SCN or mural nodule in malignant cyst, were excluded in our study populations. EUS alone may be helpful but not enough to differentiate mucinous cysts. Therefore, the addition of complementary tests such as cytology and cyst fluid analysis to EUS examination is needed.

As EUS-guided aspiration for pancreas cyst fluid became possible, cyst fluids have been examined for several years. In many studies, cyst fluid CEA is well known to be the most useful test for diagnosis of mucinous cyst; however, the reference values are different for each study. One large multicenter study in 2004 reported that the optimal cutoff value of cyst fluid CEA for differentiating mucinous versus nonmucinous cystic lesions was $192 \mathrm{ng} / \mathrm{mL}$. ${ }^{9}$ Subsequent study in 2011 showed that cyst fluid CEA had an accuracy of 86\% when an optimal cutoff value of $109.9 \mathrm{ng} / \mathrm{mL}$ was use to predict mucinous cyst. ${ }^{19}$ Other meta-analysis including twelve studies which comprised data of 450 patients reported that CEA $<5 \mathrm{ng} / \mathrm{mL}$ suggested serous-cystadenoma or pseudocyst and CEA $>800 \mathrm{ng} / \mathrm{mL}$ would strongly indicate mucinous-cystadenoma or mucinous cystadenocarcinoma. ${ }^{20}$ Many other studies at various centers have reported a wide range of diagnostic CEA cutoff values from 30 to $480 \mathrm{ng} / \mathrm{mL}$ for the diagnosis of mucinous cyst. ${ }^{20-24}$ There are many reasons about these diverse CEA values for the diagnosis of mucinous cyst. First, it might be due to the fact that subjects used in various studies are different. Some literatures included IPMN, whereas others excluded IPMN. ${ }^{20}$ Some studies had many malignant mucinous cysts, whereas others had few malignant cysts. The optimal cutoff value for mucinous cysts in our study was $48.6 \mathrm{ng} / \mathrm{mL}$ with sensitivity of $72.4 \%$ and specificity of 94.7\%. Our study included many IPMNs and only three malignancy patients, so it might have influenced to our results. Second, there is no validated commercially available cyst fluid CEA assay. Furthermore, the handling of cyst fluid submitted for CEA analysis is not standardized (dilution of specimen or not, kinds of fixatives, and so on). ${ }^{25}$ Therefore, different centers analyzed cyst fluid using different assay systems in their own ways. ${ }^{22-24}$ For this reason, cyst fluid CEA values using different commercial assays may not yield equivalent results. As a result, reported cutoff values are relatively center specific. It may be inappropriate to apply the same reported cutoff value to diagnose mucinous cyst in all centers. Therefore, our study suggests that each centers or countries may require its own adjusted cutoff value of cyst fluid CEA.

It is reported that cytology has low sensitivity. The overall cytologic accuracy of EUS-FNA for pancreas cyst is only about 50\%. ${ }^{9,26,27}$ Aspirated fluid usually has too low cellularity to provide sufficient cellular components for diagnosis. Therefore, the role of EUS-FNA for pancreas cystic lesion is limited unlike for pancreas solid mass. As a result, cytology is often nondiagnostic. ${ }^{28}$ Our study also demonstrated that a low yield of cytology when used alone for diagnosing mucinous cysts, with a shown sensitivity of only $24.1 \%$. However, it showed a high specificity of 100\%. Three mucinous cysts that had been considered as nonmucinous cyst by using fluid CEA cutoff value (48.6 ng/mL) could be identified as mucinous cysts by cytology. Therefore, cytologic examination is an important diagnosis method of mucinous cysts in spite of its low sensitivity.

Mucinous cysts usually contain highly viscous clear fluid. Therefore, acquisition of enough amount of samples for diagnosis may be difficult by aspiration. String sign can be done by measuring the maximal length for both end of the mucus string before disruption when cyst fluid is placed to examiner's the thumb and index finger and stretched. One study reported that the median string sign was $0 \mathrm{~mm}$ in benign cysts and 3.5 $\mathrm{mm}$ in mucinous cysts. ${ }^{29}$ We did not check the length of mucus string. We only discriminated positive or negative for string sign (standard $3 \mathrm{~mm}$ ). In our study, although it showed relative low sensitivity at 53.6\%, it showed 100\% specificity In addition, it increased the accuracy when it was combined with cytology and cyst fluid CEA. Five cases of mucinous cysts had been considered as nonmucinous cysts by using cyst fluid CEA criteria, but they could be diagnosed as mucinous cysts by using string sign. String sign can be used as a complementary method because it is a cost-effective method that can be performed easily and quickly for the diagnosis of mucinous cyst. A total of six patients had mucinous cysts with positive string sign or cytology and negative CEA level (below cutoff value $48.6 \mathrm{ng} / \mathrm{mL}$ ) in our study. Three patients had mucinous cysts with only positive string sign and one patient had mucinous cyst with only positive cytology and two patients had mucinous cysts with both tests positive. Among them, three patients underwent surgery due to increase in size and three patients underwent surgery due to IPMN with worrisome feature or high risk stigmata on EUS.

Many previous studies reported about EUS findings, cyst fluid analysis, cytology for diagnosis of mucinous cyst. But there were few reports about viscosity test and also there were no report about combination test of these methods as far as we know. Also, in a large multicenter trial, the combination test of EUS morphology and cytology with cyst fluid CEA did not increase 
the accuracy compared to using cyst fluid CEA concentration alone. ${ }^{9}$ However, our study showed that the combination of cytology, string sign, and cyst fluid CEA increased the sensitivity with equivalent specificity. In addition, the combination provided additional diagnostic accuracy. Therefore, our study results suggest that combination of the three tests would be helpful in patient with pancreas cyst which are difficult to determine the treatment plan (operation or follow-up) in real clinical practice.

Our study has some limitations. First, this was a retrospective study at a single tertiary medical center in Korea. Endosonographers were not blinded to the results of prior imaging studies, which might have influenced EUS results. Second, selection bias may be present in this study. Most patients who were analyzed in this study composed of patients who underwent surgical resection due to many reasons including presumed mucinous cysts, symptoms, increasing size and so on. The characteristics of these patients may be different from an unselected population. Therefore, our results may not be applicable to all patients with pancreatic cystic lesion. However, our patient group might be similar to subjects who need to do EUS-FNA and cyst fluid analysis in real clinical practice. Third, our study involved a small sample size. It could influence the cyst fluid CEA cutoff value and statistical significance. Therefore, collecting additional cases from large multicenters is needed to validate our findings in the future.

In conclusion, this investigation assessed the usefulness of EUS imaging and diagnostic accuracy of cyst fluid CEA, cytology, string sign, and the combination of the three for differentiating mucinous versus nonmucinous pancreatic lesions. In our study, the optimal cutoff value of cyst fluid CEA for differentiating mucinous cystic lesions was $48.6 \mathrm{ng} / \mathrm{mL}$. We suggest that adjusted CEA cutoff value may be needed for each centers or countries. The sensitivity of combination test was 93.1\%, more sensitive than one single method. Also a combination test using cyst fluid CEA, cytology, and string sign could increase the diagnostic accuracy 93.8\% than using cyst fluid CEA alone for the diagnosis of mucinous cystic lesions.

\section{CONFLICTS OF INTEREST}

No potential conflict of interest relevant to this article was reported.

\section{REFERENCES}

1. Spinelli KS, Fromwiller TE, Daniel RA, et al. Cystic pancreatic neoplasms: observe or operate. Ann Surg 2004;239:651-657.

2. Kimura W, Nagai H, Kuroda A, Muto T, Esaki Y. Analysis of small cystic lesions of the pancreas. Int J Pancreatol 1995;18:197-206.

3. Ferrone CR, Correa-Gallego C, Warshaw AL, et al. Current trends in pancreatic cystic neoplasms. Arch Surg 2009;144:448-454.

4. Longnecker DS, Hruban RH, Adler G, Klöppel G. Intraductal papillary-mucinous neoplasm. In: Hamilton SR, Aaltonen LA, eds. World Health Organization classification of tumours: pathology and genetics of tumours of the digestive system. Lyon: IARC Press, 2000:237-241.

5. Balthazar EJ, Chako AC. Computed tomography of pancreatic masses. Am J Gastroenterol 1990;85:343-349.

6. Wolfman NT, Ramquist NA, Karstaedt N, Hopkins MB. Cystic neoplasms of the pancreas: CT and sonography. AJR Am J Roentgenol 1982;138:37-41.

7. Johnson CD, Stephens DH, Charboneau JW, Carpenter HA, Welch TJ. Cystic pancreatic tumors: CT and sonographic assessment. AJR Am J Roentgenol 1988;151:1133-1138.

8. Friedman AC, Lichtenstein JE, Dachman AH. Cystic neoplasms of the pancreas: radiological-pathological correlation. Radiology 1983;149:45-50.

9. Brugge WR, Lewandrowski K, Lee-Lewandrowski E, et al. Diagnosis of pancreatic cystic neoplasms: a report of the cooperative pancreatic cyst study. Gastroenterology 2004;126:1330-1336.

10. Fisher WE, Hodges SE, Yagnik V, et al. Accuracy of CT in predicting malignant potential of cystic pancreatic neoplasms. HPB (OXford) $2008 ; 10: 483-490$.

11. Visser BC, Yeh BM, Qayyum A, Way LW, McCulloch CE, Coakley FV. Characterization of cystic pancreatic masses: relative accuracy of CT and MRI. AJR Am J Roentgenol 2007;189:648-656.

12. Gress F, Gottlieb K, Cummings O, Sherman S, Lehman G. Endoscopic ultrasound characteristics of mucinous cystic neoplasms of the pancreas. Am J Gastroenterol 2000;95:961-965.

13. Ahmad NA, Kochman ML, Lewis JD, Ginsberg GG. Can EUS alone differentiate between malignant and benign cystic lesions of the pancreas? Am J Gastroenterol 2001;96:3295-3300.

14. Koito K, Namieno T, Nagakawa T, Shyonai T, Hirokawa N, Morita K. Solitary cystic tumor of the pancreas: EUS-pathologic correlation. Gastrointest Endosc 1997;45:268-276.

15. Palazzo L, Roseau G, Gayet B, et al. Endoscopic ultrasonography in the diagnosis and staging of pancreatic adenocarcinoma: results of a prospective study with comparison to ultrasonography and CT scan. Endoscopy 1993;25:143-150.

16. Müller MF, Meyenberger C, Bertschinger P, Schaer R, Marincek B. Pancreatic tumors: evaluation with endoscopic US, CT, and MR imaging. Radiology 1994;190:745-751.

17. Hammel P, Levy P, Voitot H, et al. Preoperative cyst fluid analysis is useful for the differential diagnosis of cystic lesions of the pancreas. Gastroenterology 1995;108:1230-1235.

18. Young NA, Villani MA, Khoury P, Naryshkin S. Differential diagnosis of cystic neoplasms of the pancreas by fine-needle aspiration. Arch Pathol Lab Med 1991;115:571-577.

19. Cizginer S, Turner BG, Bilge AR, Karaca C, Pitman MB, Brugge WR. Cyst fluid carcinoembryonic antigen is an accurate diagnostic marker of pancreatic mucinous cysts. Pancreas 2011;40:10241028.

20. van der Waaij LA, van Dullemen HM, Porte RJ. Cyst fluid analysis in the differential diagnosis of pancreatic cystic lesions: a pooled 
analysis. Gastrointest Endosc 2005;62:383-389.

21. Snozek CL, Mascarenhas RC, O'Kane DJ. Use of cyst fluid CEA, CA19-9, and amylase for evaluation of pancreatic lesions. Clin Biochem 2009;42:1585-1588.

22. Linder JD, Geenen JE, Catalano MF. Cyst fluid analysis obtained by EUS-guided FNA in the evaluation of discrete cystic neoplasms of the pancreas: a prospective single-center experience. Gastrointest Endosc 2006;64:697-702.

23. Attasaranya S, Pais S, LeBlanc J, McHenry L, Sherman S, DeWitt JM. Endoscopic ultrasound-guided fine needle aspiration and cyst fluid analysis for pancreatic cysts. JOP 2007;8:553-563.

24. Shami VM, Sundaram V, Stelow EB, et al. The level of carcinoembryonic antigen and the presence of mucin as predictors of cystic pancreatic mucinous neoplasia. Pancreas 2007;34:466-469.

25. Pitman MB, Lewandrowski K, Shen J, Sahani D, Brugge W, Fernandez-del Castillo C. Pancreatic cysts: preoperative diagnosis and clinical management. Cancer Cytopathol 2010;118:1-13.

26. Frossard JL, Amouyal P, Amouyal G, et al. Performance of endosonography-guided fine needle aspiration and biopsy in the diagnosis of pancreatic cystic lesions. Am J Gastroenterol 2003;98:1516-1524.

27. Wiersema MJ, Vilmann P, Giovannini M, Chang KJ, Wiersema LM. Endosonography-guided fine-needle aspiration biopsy: diagnostic accuracy and complication assessment. Gastroenterology 1997;112:1087-1095.

28. Centeno BA, Warshaw AL, Mayo-Smith W, Southern JF, Lewandrowski K. Cytologic diagnosis of pancreatic cystic lesions: a prospective study of 28 percutaneous aspirates. Acta Cytol 1997;41:972-980.

29. Leung KK, Ross WA, Evans D, et al. Pancreatic cystic neoplasm: the role of cyst morphology, cyst fluid analysis, and expectant management. Ann Surg Oncol 2009;16:2818-2824. 